\title{
GAIA Level 1 Vasa Previa
}

National Cancer Institute

\section{Source}

National Cancer Institute. GAIA Level 1 Vasa Previa. NCI Thesaurus. Code C128746.

GAIA Level 1 Vasa Previa is defined by two criteria: first, second trimester ultrasound evidence of fetal vessels (vessel with fetal heart rate identified by color flow Doppler) traversing the membranes and overlying the internal cervical os; second, post-delivery examination of the placental specimen that demonstrates unsupported fetal vessels within the membranes. 